\title{
Progressive pseudorheumatoid dysplasia confirmed by whole-exon sequencing in a Chinese adult before corrective surgery
}

\author{
Yan Li ${ }^{1}$, Yan Zeng ${ }^{1 *} \mathbb{D}$, Zhongqiang Chen ${ }^{1}$, Haisong $\mathrm{Xin}^{2}$ and Xiaoliang $\mathrm{Li}^{2}$
}

\begin{abstract}
Background: Progressive pseudorheumatoid dysplasia (PPD) is a rare autosomal recessive skeletal dysplasia caused by mutations in the Wnt1-inducible signaling pathway protein 3 (WISP3) gene. Available literatures in PPD emphasized treatment strategy for polyarthritis, while few mentioned spinal deformity and related surgical intervention.

Methods: Here, we present a Chinese man with PPD who underwent spinal surgery twice because of canal stenosis and related symptoms caused by the disease. Whole-exon sequencing (WES) was performed to confirm diagnosis before the second surgery.

Results: A homozygous missense mutation (c.395G>A/p.C132Y) in WISP3 was identified that co-segregated with affected family members.

Conclusions: Our study illustrated a surgical outcome of PPD and highlighted the significance of early diagnosis and individualized surgical strategy, and also verified the value of WES in the diagnosis of PPD.
\end{abstract}

Keywords: Progressive pseudorheumatoid dysplasia, Whole-exon sequencing, Spinal surgery

\section{Background}

Progressive pseudorheumatoid dysplasia (PPD) is an autosomal recessive disease characterized by spondyloepiphyseal dysplasia associated with progressive joint deformities, pain, stiffness, and swelling mainly in the spine and hip joints [1]. PPD is caused by mutation in the Wnt1-inducible signaling pathway protein 3 (WISP3) gene, which consists of five exons and encodes a 354 amino acid protein [2]. WISP3 belongs to the connective tissue growth factor $(\mathrm{CCN})$ gene family, and the encoded protein plays an essential role in skeletal growth and cartilage homeostasis [3]. This disease is usually misdiagnosed as juvenile idiopathic arthritis or juvenile rheumatoid arthritis (JRA), and patients can receive many years of unnecessary treatment before correct diagnosis [4].

Whole-exon sequencing (WES) is useful for identifying rare monogenic inherited diseases [5], and the reducing

\footnotetext{
*Correspondence: prof_yanzeng@163.com

'Department for Orthopedics, Peking University Third Hospital, Huayuanbei Rd 49\#, Beijing 100191, China

Full list of author information is available at the end of the article
}

cost of WES has improved the feasibility of its clinical use [6]. In this study, we employed WES to explore the potential causative genes in a Chinese PPD patient who underwent spinal surgical treatment twice.

\section{Methods \\ Case presentation}

The proband was a 35-year-old male from Hebei province in the north of China. He is of Han ethnicity and was born to consanguineous parents. His family pedigree is shown in Fig. 1. The proband was normally delivered after a full-term pregnancy, and birth weight and length were within normal ranges. The initial signs and symptoms appeared when he was 6 years old. Deformity of interphalangeal joints initially appeared in the fingers. Hips, knees, and wrists were then gradually involved. Diagnosis of JRA was considered by local hospitals, and glucocorticoids were prescribed without any efficacy. As he grew up, his symptoms deteriorated. He had to walk with crutches at 16 years of age because of arthritis of the lower extremities. At 26 years of age, he first 


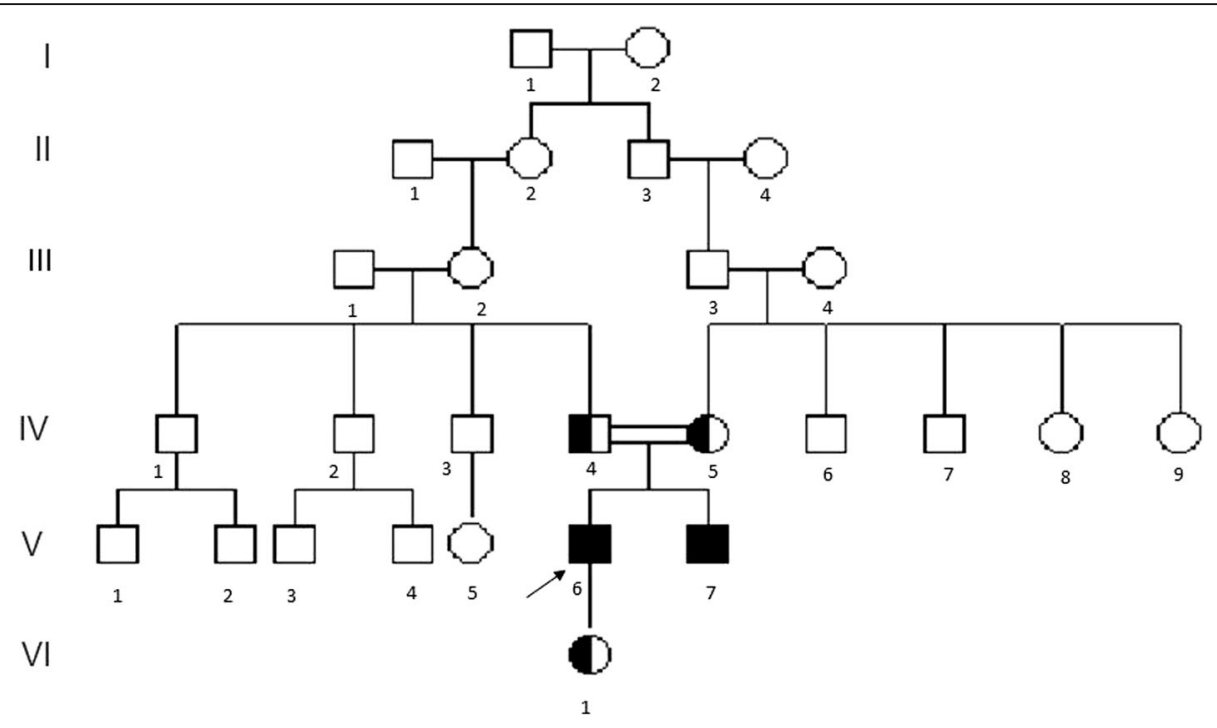

Fig. 1 Pedigree of the studied Chinese family with progressive pseudorheumatoid dysplasia, presenting co-segregation of the c.395G $>$ A mutation. The patient indicated by the arrow is the proband

experienced progressive pain with numbness radiating down his entire left leg and right thigh. At 34, he started to have mild difficulty in urination. Thereafter, his leg pain progressed and he became immobile. Treatment with tramadol, physical therapy, and spine injection were tried but were not effective. He had a younger brother with a similar clinical presentation but who also had mild neurological impairment (Fig. 2).

The proband's height and weight were $162 \mathrm{~cm}$ and $72.5 \mathrm{~kg}$ when he was admitted to our hospital. His visual analogue scale (VAS) score was 9. He did not have behavioral difficulties and was not retarded in his intellectual development. Physical examination showed multiple malformations of the major limb joints, especially of the knees and hands (Fig. 2). Amyotrophy of both lower limbs was obvious. Cervical and lumbar movements were limited with compensatory kyphosis. The muscular strength of all four limbs was normal. Dysesthesia was found in the posterolateral left calf, dorsolateral left foot, and perineal area. Bilateral knee-jerk reflexes and ankle reflexes were hypo-induced. The erythrocyte sedimentation rate $(13 \mathrm{~mm} / \mathrm{h})$ and $\mathrm{C}$-reactive protein level $(2 \mathrm{mg} / \mathrm{L})$ were both within the normal range. Tests for rheumatoid factors were negative.

Spinal $\mathrm{x}$-rays showed flat and osteoporotic vertebral bodies. Pedicles were short, and end plates were irregular. Bone bridges were seen at many levels. Kyphosis was detected in both the cervical and upper thoracic spine. Magnetic resonance imaging showed multilevel Schmorl nodes. Multilevel disc herniation and hypertrophic ligamentum flavum caused lumbar canal stenosis from L2 to S1 (Fig. 3).

\section{DNA extraction}

Blood samples were taken from the proband and from direct consanguineous relatives from three generations after an informed consent was obtained. DNA was extracted using a centrifuge column method (Tiangen, Beijing, China) and was qualitatively and quantitatively assessed by standard techniques before use in wholeexome sequencing.

\section{Whole-exon sequencing}

All exons were captured using the Roche NimbleGen human exon V2 capture chip (Roche, Pleasanton, CA, USA) according to the manufacturer's protocols, and sequencing data was obtained using the Roche NimbleGen human exon V2 capture chip on the Illumina HiSeq 2500 platform (Illumina Inc.).

The data filtering strategies were as follows: To identify reliable variations, those with a sequencing depth of at least 20 and a variation depth/total depth ratio higher than $20 \%$ were included. Because we were looking for rare mutations, those with minor allele frequency (MAF) higher than $0.1 \%$ in the 1000 Genomes Project and ExAc database were removed. Variants in the dbSNP 144 with MAF higher than $0.1 \%$ were also excluded. Synonymous and non-functional changes were also excluded. Deleterious variants located in the coding area of genes that were functionally related to skeletal dysplasia (Online Mendelian Inheritance in Man (OMIM), https://www.ncbi.nlm. nih.gov/omim) were selected using both PolyPhen-2 (http://genetics.bwh.harvard.edu/pph2/) and SIFT (http:// sift.jcvi.org/). The procedure is described in Fig. 4. 

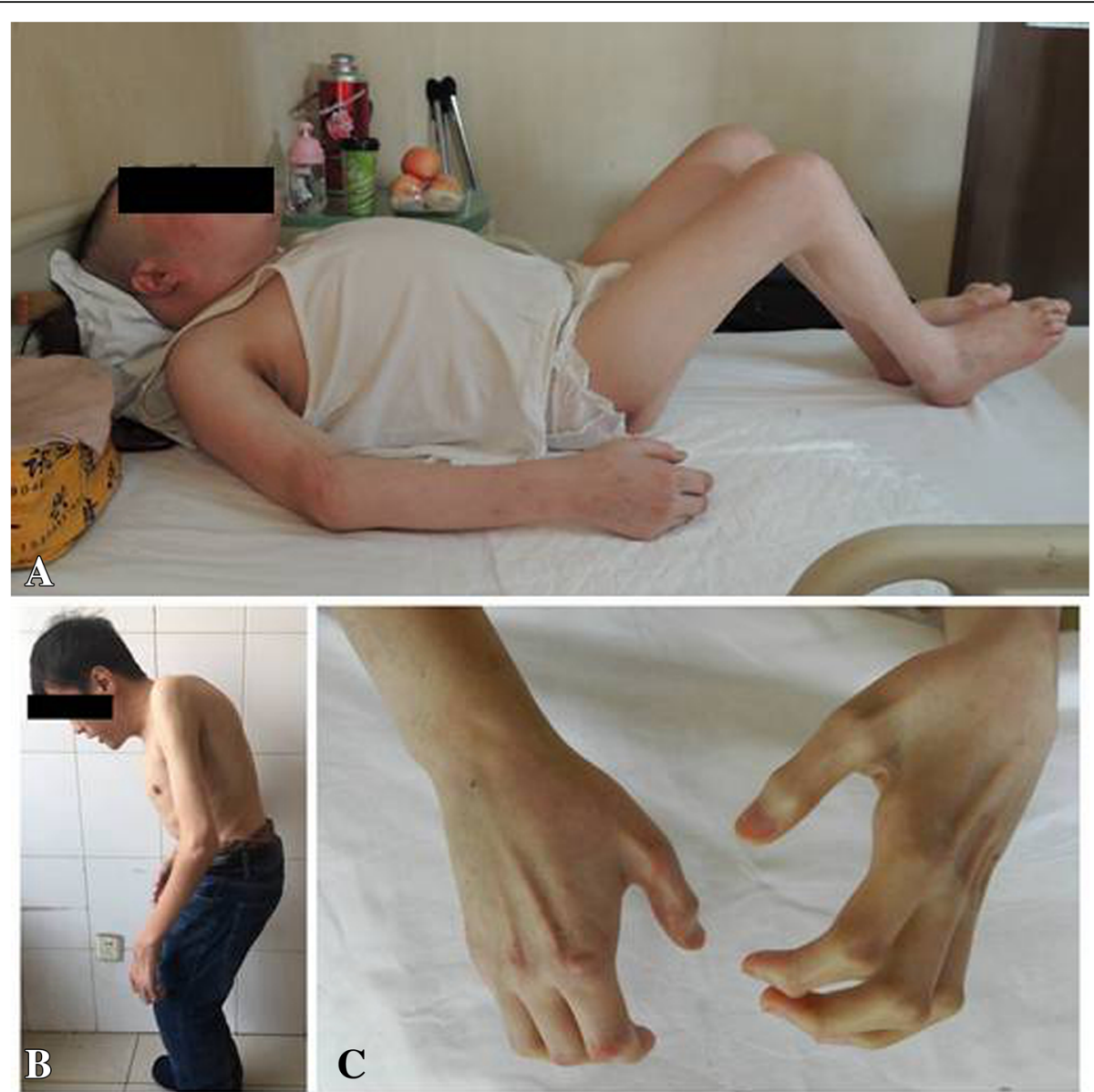

Fig. 2 General views of the proband (a) and his younger brother (b) show kyphosis of the spine and multiple deformities of the extremities. The hands of the proband were initially involved, and their motion range was constrained by deformity (c)

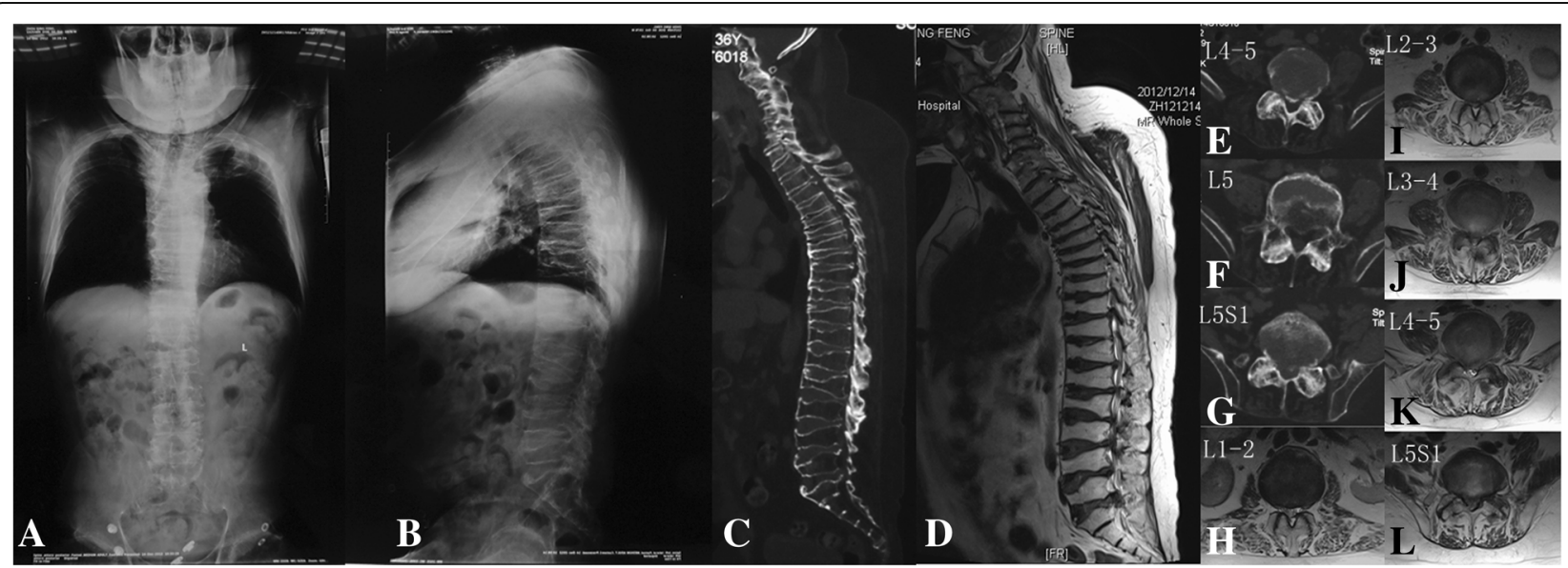

Fig. 3 X-ray and CT scan of the whole spine showed osteoporotic flat vertebral bodies and bone bridges at multiple levels (a-c). Axial CT scan revealed maldevelopment of the left pedicle of $L 5$ (f). Ossified herniated discs at L4-5 and L5-S1 were showed $(\mathbf{c}, \mathbf{e}, \mathbf{g})$. Magnetic resonance imaging showed extensively canal stenosis from L2 to S1 (d, $\mathbf{h}-\mathbf{l})$ 
A

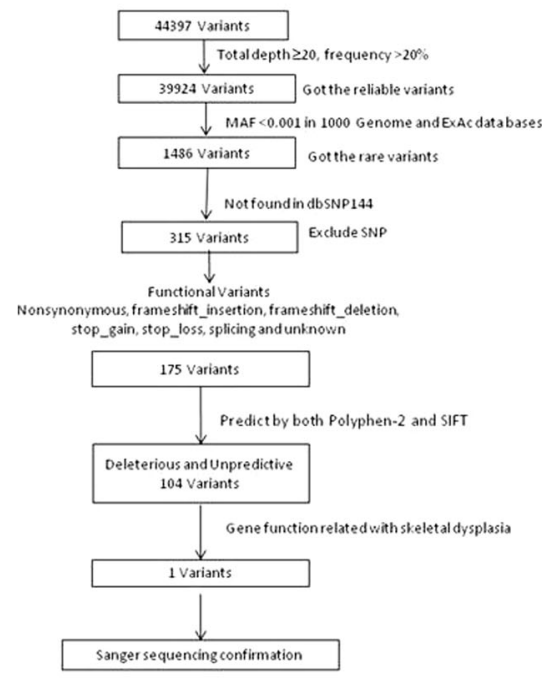

B

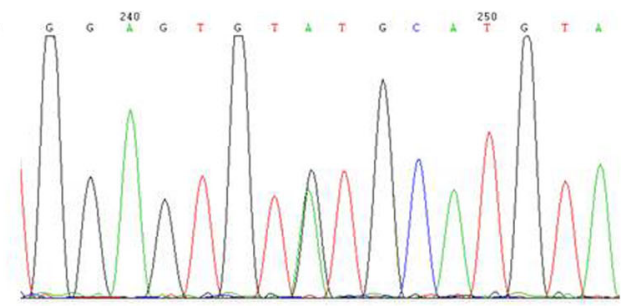

C

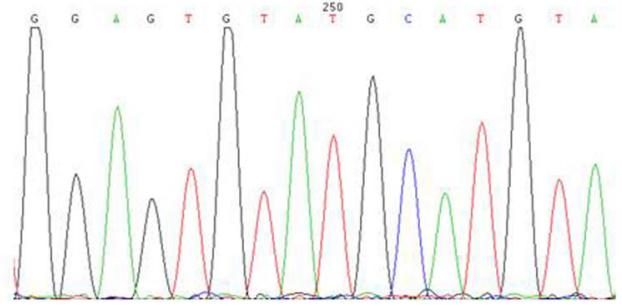

D

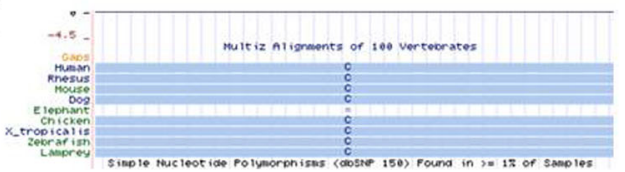

Fig. 4 The data screening process of whole-exon sequencing revealed variants (a). Sanger sequencing chromatograms of family members who are heterozygous (b) and homozygous (c) for the WISP3 variant. Amino acid conservation map across species demonstrating a highly conserved residue at position 132 (d, based on Multiz Alignments Track of UCSC Genome Browser and the human genome reference hg19/GRCch37)

\section{Segregation analysis}

The putative mutation was validated by Sanger sequencing in the proband and four family members. The following PCR primers were designed using Primer 6.0 software (PREMIER Biosoft International, CA, USA): forward (CAGGGCACTGGACCATTAGA) and reverse (CCCACTGGTGCATGAAAACTAA). PCR products were purified and sequenced on an ABI 3130XL (Applied Biosystems). The results are shown in Fig. 4.

\section{Results}

At the first admittance to our hospital, the diagnosis of the proband was not clear. Mucolipidosis type IV was suspected according to clinical presentations. Both physical examination and imaging indicated lumbar canal stenosis at that time, so we performed decompressive laminectomy and posterior-lateral fusion from L2 to S1. A pedicle screw internal fixation system was used (Synthes GmbH, USS II, Switzerland Inc.; Fig. 5), but the left pedicle of L5 was maldeveloped making it impossible to implant a screw (Fig. 3). Hypertrophic ligamentum flavum and herniated discs from L2 to S1 were confirmed during the surgery. The herniated discs of L4-5 and L5-S1 were ossified which caused cauda equina compression (Fig. 3). The dura at the L5-S1 level was hypertrophic and rigid. After the operation, leg pain was decreased and the VAS score was decreased to 1 without any surgical complications. With the help of rehabilitation training, the proband regained the ability to walk with the help of crutches.
Bony fusion of the facet joints from L2 to S1 was achieved in the follow-up (Fig. 5).

Five years after the surgery, the patient began to feel stiffness and weakness of the lower extremities. His symptoms progressed for several months, and he lost ambulatory ability again. Physical examination revealed positive Babinski sign, hypesthesia below the xiphoid level, hypermyotonia, and extensive muscle weaknesses of the lower extremities. Magnetic resonance imaging showed extensive canal stenosis from T2 to L2 (Fig. 6). Kyphosis of the middle thoracic spine increased compression of the spinal cord, and Frankel classification was grade B. The proband underwent surgery again, during which decompressive laminectomy from T2 to L2 was performed and kyphosis correction was achieved by resecting the facet joints with circumferential decompression at the T5-6 level. We also removed the L2 pedicle screws and connected the segmental instrumentation (Synthes GmbH, USS II, Switzerland Inc.) with the previous internal fixation. Zonesthesia and hypermyotonia of the lower extremities improved postoperatively, but muscle weakness did not improve. At the latest follow-up, 1 year after the second surgery, the Frankel classification was still grade B.

Before the second surgery, WES was performed and a conclusive diagnosis of PPD was developed. WES covered $99.8 \%$ of target regions with a mean depth of ontarget reads of $\times 105$. A total of 44,397 raw variant calls were detected, and after removing variants with low 


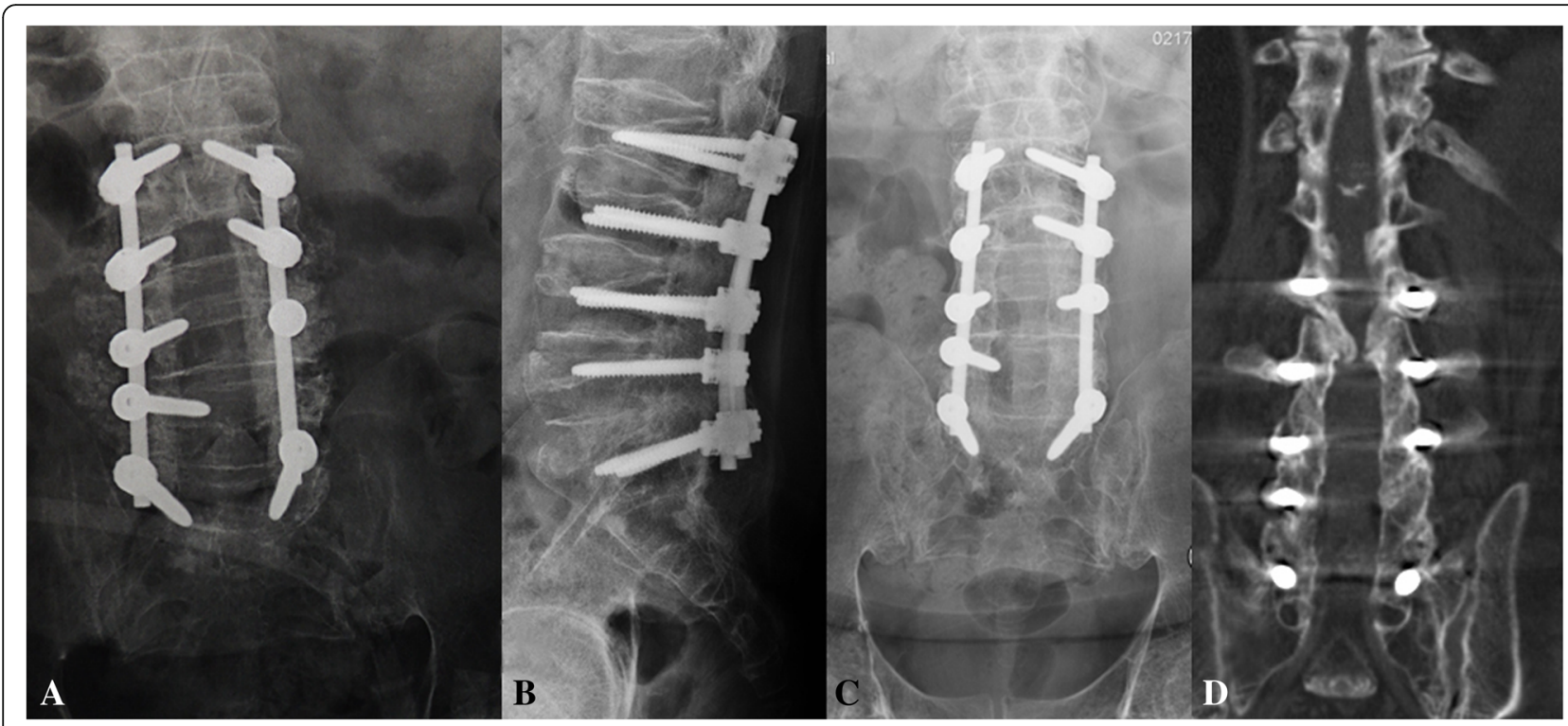

Fig. 5 Extensive decompression of lumbar canal with internal fixation from L2 to S1 was implemented during the first surgery (a). X-ray and CT images suggest facet joint fusion 5 years later $(\mathbf{b}-\mathbf{d})$

quality (see the "Methods" section), 39,924 remained. Using the 1000 Genomes and ExAc databases, 1486 were selected as rare variants, $79.0 \%$ of which were excluded as known SNPs. Also excluded were 140 synonymous variants, while 71 benign or neutral variants were filtered out by PolyPhen-2 and SIFT. One rare variant (c.395G $>$ A/p.C132Y) located in exon 3 of WISP3 was identified as associated with skeletal dysplasia. Sanger sequencing confirmed this homozygous mutation co-segregated with affected family members (Fig. 4). This mutation resulted in the substitution of a cysteine with a tyrosine at position 132 and was predicted by Polyphen- 2 as probably damaging with a score of 1.0 and damaging by SIFT with a score of 0 . As shown in Fig. 4, this amino acid change affected a highly conserved residue.

\section{Discussion}

PPD is a rare autosomal recessive disease, with an estimated population incidence in the UK of one per million [1]. In this study, we presented a family showing PPD and a patient undergoing spinal surgery twice. WES identified a missense mutation in WISP3, enabling a definitive diagnosis. We believe that this is the first presentation of a PPD patient receiving spinal surgery twice.

Patients with PPD are asymptomatic after birth and during early childhood, with symptoms often appearing between the age of 4 and 6 years [7, 8]. Because PPD patients are asymptomatic in the first years of life, the disease is usually misdiagnosed as mucolipidosis type IV and juvenile rheumatoid arthritis [9]. The typical clinical manifestations and radiographic findings in PPD include progressive deformities, pain, stiffness, and swelling of

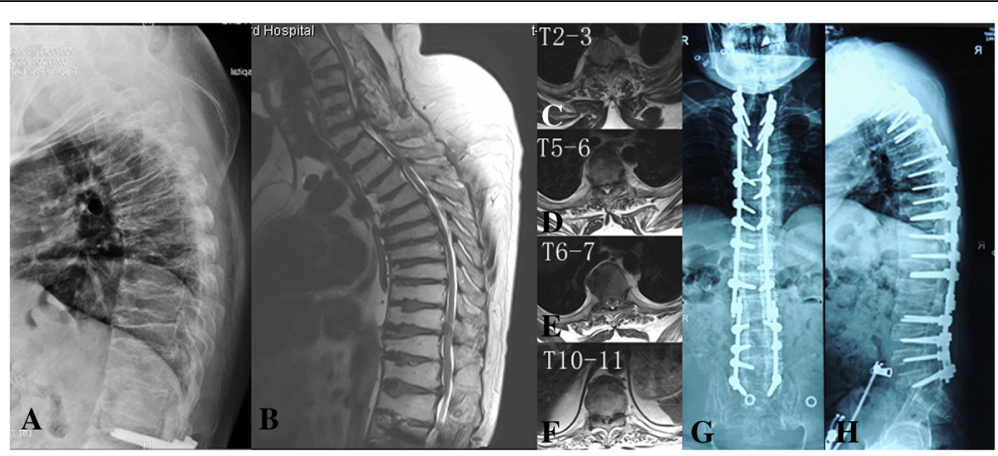

Fig. 6 Before the second surgery, x-ray and magnetic resonance imaging of the thoracic spine showed kyphosis and multilevel canal stenosis $(\mathbf{a}-\mathbf{f})$. During the second surgery, we extensively decompressed the spinal canal from T2 to L2 and mildly corrected kyphosis of the middle thoracic spine $(\mathbf{g}, \mathbf{h})$ 
multiple joints, notably in the wrists, fingers, hips, and knees, with the absence of destructive bone changes [7]. To date, there is still no radical treatment for PPD and surgical treatment can be performed in individuals who might benefit. In our patient, the hypogenetic spine presented coin-like flat vertebral bodies, short pedicles, and kyphosis. These deformities resulted in developmental spinal canal stenosis. The degenerative changes, such as disc herniation and hypertrophic ligamentum flavum, worsened the spinal canal stenosis and gradually led to clinical symptoms. Spinal surgery aiming to decompress the spinal canal was inevitable if non-surgical intervention failed. Our patient also had developmental spinal canal stenosis and conspicuous spinal symptoms. He underwent lumbar spine and thoracic spine surgery successively. Available reports on the treatment for PPD focus more on hip joint replacement surgery, and this is the first reported sequential lumbar and thoracic spinal surgery. Meanwhile, vertebral body deformities, maldevelopment of the pedicles and segmental kyphosis, increased the difficulty of the spinal surgery. We noticed that muscle weakness did not improve after the second surgery, indicating the importance of early diagnosis and rehabilitation intervention.

WISP3 belongs to the $\mathrm{CCN}$ family, which encodes multimodular mosaic proteins. To date, 51 mutations have been identified in WISP3, including missense, nonsense, frameshift, and exon-deletion mutations (the $\mathrm{Hu}-$ man Gene Mutation Database (HGMD)), indicating that loss of WISP3 function leads to PPD. Our study is consistent with another report of a Chinese patient with a similar phenotype and a WISP3 p.C132Y mutation [10], which indicates this variant as a mutation hotpoint in the Chinese population. WISP3 functions in the synthesis of chondrocyte saccharin and collagen type II [11], which are the major components of cartilaginous tissue. The loss of WISP3 function can result in cartilage lesion.

WES enabled us to conclusively diagnose PPD in this patient and may provide wider clinical use in this field. With its decreasing cost, WES is increasingly used for diagnostic purposes in the clinical setting with a $25.2 \%$ diagnosis rate in a consecutive patient cohort [5]. With appropriate data filtering and pedigree mapping, WES can help develop diagnoses, especially in rare Mendelian diseases like PPD, which will help prevent the administration of unnecessary treatments [12]. Our study further verified the value of WES in the diagnosis of PPD. With the diagnosis of PPD, we were able to develop proper surgical strategy and anticipate complexity of surgery before the second procedure. Due to coin-like flat vertebral bodies of PPD, precisely applying pedicle screw should be pre-designed before surgery [13]. And since developmental spinal canal stenosis was induced by short pedicles in PPD, associated cervical, thoracic, and lumbar neurological symptoms may deteriorate over time with degenerative changes. As a result, spinal decompression range should be decided not only on the radiological imaging, but also on clinical manifestations. Favorable prognosis in PPD patients was also composed by patient education in early intervention of myelopathy and anti-osteoporosis medications.

\section{Conclusions}

In conclusion, our study describes a PPD patient who underwent spinal surgery twice. Meanwhile, WES was used efficiently to identify causative mutations in PPD, thus informing a definitive diagnosis and enabling individualized surgical strategy.

\section{Abbreviations}

HGMD: Human Gene Mutation Database; JRA: Juvenile rheumatoid arthritis; MAF: Minor allele frequency; OMIM: Online Mendelian Inheritance in Man; PPD: Progressive pseudorheumatoid dysplasia; VAS: Visual analogue scale; WES: Whole-exon sequencing; WISP3: Wnt1-inducible signaling pathway protein 3

\section{Acknowledgements}

The authors are indebted to the individuals whose participation made this study possible. We would like to acknowledge Dr. Wei Liu and Dr. Siqian Gong (both from Peking University People's Hospital) for their generous help with gene mutation analysis.

\section{Funding}

This research was supported by Natural Science Foundation of Beijing Municipality (A73509-03).

\section{Availability of data and materials}

Datasets used in this study are available from the corresponding author.

\section{Authors' contributions}

YL initiated the idea, did the data analysis, and wrote the assay. YZ supervised and reviewed the manuscript, and also provided the funding. ZC supervised the study. HX and XL gathered the data and helped with the data analysis. All authors read and approved the final manuscript.

Ethics approval and consent to participate

This study was approved by the Peking University Third Hospital Ethical Committee and taken out under the direction of Declaration of Helsinki. Written consents were obtained from all the participants.

\section{Consent for publication}

Consent for publication had been obtained from the participants.

Competing interests

The authors declare that they have no competing interests.

\section{Publisher's Note}

Springer Nature remains neutral with regard to jurisdictional claims in published maps and institutional affiliations.

\section{Author details}

'Department for Orthopedics, Peking University Third Hospital, Huayuanbei Rd 49\#, Beijing 100191, China. ${ }^{2}$ Department for Orthopedics, People's Hospital of Huanghua, Cangzhou, Hebei, China. 
Received: 3 May 2018 Accepted: 3 January 2019

Published online: 11 January 2019

\section{References}

1. Wynne-Davies R, Hall C, Ansell BM. Spondylo-epiphysial dysplasia tarda with progressive arthropathy. A "new" disorder of autosomal recessive inheritance. J Bone Joint Surg. 1982;64:442-5.

2. Hurvitz JR, Suwairi WM, Van Hul W, El-Shanti H, Superti-Furga A, Roudier J, Holderbaum D, Pauli RM, Herd JK, Van Hul EV, Rezai-Delui H, Legius E, Le Merrer M, Al-Alami J, Bahabri SA, Warman ML. Mutations in the CCN gene family member WISP3 cause progressive pseudorheumatoid dysplasia. Nat Genet. 1999;23:94-8.

3. Sen M, Cheng YH, Goldring MB, Lotz MK, Carson DA. WISP3-dependent regulation of type II collagen and aggrecan production in chondrocytes. Arthritis Rheum. 2004:50:488-97.

4. Garcia Segarra N, Mittaz L, Campos-Xavier AB, Bartels CF, Tuysuz B, Alanay Y, Cimaz R, Cormier-Daire V, Di Rocco M, Duba HC, Elcioglu NH, Forzano F, Hospach T, Kilic E, Kuemmerle-Deschner JB, Mortier G, Mrusek S, Nampoothir S, Obersztyn E, Pauli RM, Selicorni A, Tenconi R, Unger S, Utine GE, Wright M, Zabel B, Warman ML, Superti-Furga A, Bonafé L. The diagnostic challenge of progressive pseudorheumatoid dysplasia (PPRD): a review of clinical features, radiographic features, and WISP3 mutations in 63 affected individuals. Am J Med Genet C Semin Med Genet. 2012;160C:217-29.

5. Yang Y, Muzny DM, Xia F, Niu Z, Person R, Ding Y, Ward P, Braxton A, Wang M, Buhay C, Veeraraghavan N, Hawes A, Chiang T, Leduc M, Beuten J, Zhang J, He W, Scull J, Willis A, Landsverk M, Craigen WJ, Bekheirnia MR, Stray-Pedersen A, Liu P, Wen S, Alcaraz W, Cui H, Walkiewicz M, Reid J, Bainbridge M, Patel A, Boerwinkle E, Beaudet AL, Lupski JR, Plon SE, Gibbs RA, Eng CM. Molecular findings among patients referred for clinical whole-exon sequencing. JAMA. 2014;312:1870-9.

6. Monroe GR, Frederix GW, Savelberg SM, de Vries TI, Duran K, van der Smagt JJ, Terhal PA, van Hasselt PM, Kroes HY, Verhoeven-Duif NM, Nijman IJ, Carbo EC, van Gassen KL, Knoers NV, Hövels AM, van Haelst MM, Visser G, van Haaften $\mathrm{G}$. Effectiveness of whole-exon sequencing and costs of the traditional diagnostic trajectory in children with intellectual disability. Genet Med. 2016;18:949-56.

7. el-Shanti HE, Omari HZ, Qubain HI. Progressive pseudorheumatoid dysplasia: report of a family and review. J Med Genet. 1997;34:559-63.

8. Rezai-Delui H, Mamoori G, Sadri-Mahvelati E, Noori NM. Progressive pseudorheumatoid chondrodysplasia: a report of nine cases in three families. Skelet Radiol. 1994;23:411-9.

9. Nakamura Y, Weidinger G, Liang JO, Aquilina-Beck A, Tamai K, Moon RT, et al. The CCN family member Wisp3, mutant in progressive pseudorheumatoid dysplasia, modulates BMP and Wnt signaling. J Clin Invest. 2007;117:3075-86.

10. Yue H, Zhang ZL, He JW. Identification of novel mutations in WISP3 gene in two unrelated Chinese families with progressive pseudorheumatoid dysplasia. Bone. 2009;44:547-54.

11. Sen M, Cheng YH, Goldring MB, Lotz MK, Carson DA. WISP3 dependent regulation of type II collagen and aggrecan production I chondrocytes. Arthritis Rheum. 2004:50:488-97.

12. Yang Y, Muzny DM, Reid JG, Bainbridge MN, Willis A, Ward PA, Braxton A, Beuten J, Xia F, Niu Z, et al. Clinical whole-exome sequencing for the diagnosis of mendelian disorders. N Engl J Med. 2013;369:1502-11.

13. Yang $X$, Song $Y$, Kong Q. Diagnosis and surgical treatment of progressive pseudorheumatoid dysplasia in an adult with severe spinal disorders and polyarthropathy. Joint Bone Spine. 2013;80:650-2.

Ready to submit your research? Choose BMC and benefit from:
- fast, convenient online submission
- thorough peer review by experienced researchers in your field
- rapid publication on acceptance
- support for research data, including large and complex data types
- gold Open Access which fosters wider collaboration and increased citations
- maximum visibility for your research: over 100M website views per year
At BMC, research is always in progress.
Learn more biomedcentral.com/submissions

